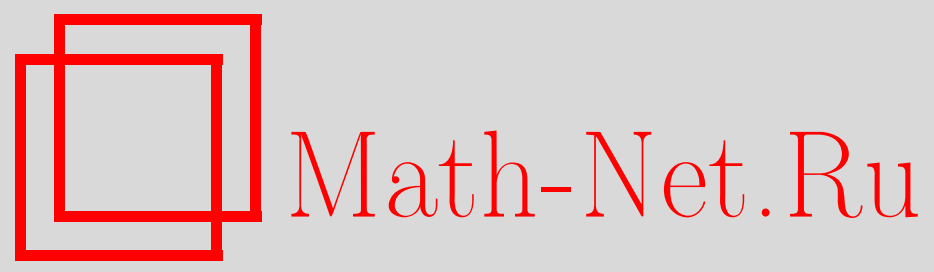

В. Г. Михайлов, Предельные теоремы для числа точек случайного линейного подпространства, попавших в заданное множество, Дискрет. матем., 2003, том 15, выпуск $2,128-137$

DOI: https://doi.org/10.4213/dm200

Использование Общероссийского математического портала Math-Net.Ru подразумевает, что вы прочитали и согласны с пользовательским соглашением http://www.mathnet.ru/rus/agreement

Параметры загрузки:

IP : 3.91 .87 .62

26 апреля 2023 г., 17:08:15

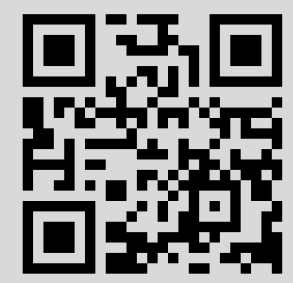




\title{
Предельные теоремы для числа точек случайного линейного подпространства, попавших в заданное множество
}

\author{
() 2003 г. В. Г. Михайлов
}

\begin{abstract}
Пусть $V^{T}-T$-мерное пространство над конечным полем $K$, заданы некоторые множества $B_{1}, \ldots, B_{m} \subseteq V^{T}$, не содержащие нулевого вектора, а подпространство $L$ выбрано случайно и равновероятно из множества всех $n$-мерных линейных подпространств пространства $V^{T}$. Рассматриваются случайные величины $\mu\left(B_{i}\right)$, равные числу точек в пересечениях $L \cap B_{i}$ при одном и том же случайно выбранном подпространстве $L, i=1, \ldots, m$. Изучается предельное поведение распределения случайного вектора $\left(\mu\left(B_{1}\right), \ldots, \mu\left(B_{m}\right)\right)$ при $T, n \rightarrow \infty$ и таком изменении множеств $B_{i}$, при котором математические ожидания величин $\mu\left(B_{i}\right)$ стремятся к конечным пределам, $i=1, \ldots, m$. Показано, что в этом случае в качестве предельного выступает сложное пуассоновское распределение. Получены условия асимптотической независимости величин $\mu\left(B_{1}\right), \ldots, \mu\left(B_{m}\right)$.

Работа выполнена при поддержке Российского фонда фундаментальных исследований, проект 02-01-00266, и Совета по грантам Президента РФ и государственной поддержке ведущих научных школ, проект 00-15-96136.
\end{abstract}

\section{1. Введение}

Рассмотрим $T$-мерное пространство $V^{T}$ над конечным полем $K$. Пусть заданы некоторые множества $B_{1}, \ldots, B_{m} \subseteq V^{T}$, не содержащие нулевого вектора, а подпространство $L$ выбрано случайно и равновероятно из множества всех $n$-мерных линейных подпространств пространства $V^{T}$. Рассмотрим случайные величины $\mu\left(B_{i}\right)$ - число точек в пересечениях $L \cap B_{i}$ при одном и том же случайно выбранном подпространстве $L, i=1, \ldots, m$. Мы исследуем предельное поведение распределения вектора $\left(\mu\left(B_{1}\right), \ldots, \mu\left(B_{m}\right)\right)$ при $T, n \rightarrow \infty$ и таком изменении множеств $B_{i}$, при котором математические ожидания величин $\mu\left(B_{i}\right)$ стремятся к конечным пределам. Считается, что при этом предельном переходе поле $K$ остается неизменным.

Начнем с ряда вспомогательных определений. Заметим, что свойство коллинеарности индуцирует на множестве $V^{T} \backslash\left\{0^{T}\right\}$ ненулевых точек $T$-мерного линейного пространства отношение эквивалентности. Классами эквивалентности для этого отношения оказываются прямые, проходящие через нулевую точку, из которых эта точка исключена. Занумеруем эти классы в некотором порядке числами от 1 до $N_{T}=\left(q^{T}-1\right) /(q-1), q=|K|$ (здесь и далее запись $|A|$ обозначает число элементов множества $A)$, и условимся обозначать их через $E_{T, 1}, \ldots, E_{T, N_{T}}$. 
Пусть $R(q, m)$ - множество $m$-мерных векторов $\bar{r}=\left(r_{1}, \ldots, r_{m}\right)$ из неотрицательных целых чисел, удовлетворяющих условиям

$$
1 \leqslant r_{1}+\ldots+r_{m} \leqslant q-1 .
$$

Для каждого $\bar{r} \in R(q, m)$ определим множество

$$
F(\bar{r})=\left\{k:\left|E_{T, k} \cap B_{i}\right|=r_{i}, i=1, \ldots, m\right\}
$$

и величины

$$
\Lambda(\bar{r})=\frac{q^{n}-1}{q^{T}}|F(\bar{r})| .
$$

Определим $N\left(a_{1}, a_{2}, a_{3}, d, B\right)$ как число решений уравнения

$$
a_{1} u^{1} \oplus a_{2} u^{2} \oplus a_{3} u^{3}=d
$$

над полем $K$ относительно тройки векторов $\left(u^{1}, u^{2}, u^{3}\right), u^{1}, u^{2}, u^{3} \in B$. Здесь $a_{1}, a_{2}, a_{3} \in K \backslash\{0\}, d \in V^{T}$. Символ $\oplus$ (и символ $\ominus$ ) мы используем как для обозначения операции сложения (вычитания) в поле $K$, так и для обозначения операции сложения (вычитания) векторов в линейных пространствах над полем $K$. Пусть

$$
N(B)=\max _{a_{1}, a_{2}, a_{3}, d} N\left(a_{1}, a_{2}, a_{3}, d, B\right) .
$$

Очевидно, что $N(B) \leqslant|B|^{2}$.

Замечание 1. В работе [6] показано, что для множеств $B$, которые являются классами смежности какой-либо подгруппы по сложению в $V^{T}$, и только для них, выполнено равенство $N(B)=|B|^{2}$.

Через $\pi_{i}\left(\lambda_{i}\right)$ (здесь в качестве индекса $i$ может выступать элемент любого конечного множества) условимся обозначать независимые в совокупности случайные величины, распределенные по закону Пуассона с указанными в скобках значениями параметра.

Теорема 1. Пусть параметры $n$ и $T$ стремятся к бесконечности, а множества $B_{1}, \ldots, B_{m} \subseteq V^{T}$ меняются так, что

$$
\begin{gathered}
\Lambda(\bar{r}) \rightarrow \lambda(\bar{r}), \quad 0 \leqslant \lambda(\bar{r})<\infty, \quad \bar{r} \in R(q, m), \\
\lambda(i)=\sum_{\bar{r} \in R(q, m)} r_{i} \lambda(\bar{r})>0, \quad i=1, \ldots, m,
\end{gathered}
$$

и для

$$
B=\bigcup_{i} B_{i}
$$

выполнено условие

$$
N(B)|B|^{-2} \rightarrow 0 .
$$

Тогда распределение случайного вектора $\left(\mu\left(B_{1}\right), \ldots, \mu\left(B_{m}\right)\right)$ сходится к распределению случайного вектора

$$
\left(\sum_{\bar{r} \in R(q, m)} r_{1} \pi_{\bar{r}}(\lambda(\bar{r})), \ldots, \sum_{\bar{r} \in R(q, m)} r_{m} \pi_{\bar{r}}(\lambda(\bar{r})) .\right.
$$

Эта сходимость равномерна для тех наборов множеств $B_{1}, \ldots, B_{m}$, которым отвечают одни и те же значения параметров $\Lambda(\bar{r})$. 
Замечание 2. Величины $\lambda(i)$ являются математическими ожиданиями предельных распределений для $\mu\left(B_{i}\right)$ в теореме 1 . Условие (4) позволяет избежать вырожденности этих распределений.

Сформулируем теперь необходимые и достаточные условия асимптотической независимости случайных величин $\mu\left(B_{1}\right), \ldots, \mu\left(B_{m}\right)$, вытекающие из теоремы 1 .

Введем обозначение $R^{\prime}(q, m)$ для множества векторов $\bar{r} \in R(q, m)$, имеющих более одной ненулевой координаты.

Следствие 1. Пусть параметры $n$ и $T$ стремятся $к$ бесконечности, а множества $B_{1}, \ldots, B_{m} \subseteq V^{T}$ меняются так, что найдутся такие постоянные $C_{1}, C_{2}$, что

$$
0<C_{1} \leqslant \sum_{\bar{r} \in R(q, m)} r_{i} \Lambda(\bar{r}) \leqslant C_{2}<\infty, \quad i=1, \ldots, m,
$$

$u \partial л я$

$$
B=\bigcup_{i} B_{i}
$$

выполнено условие (5).

Тогда условие, состоящее в том, что

$$
\Lambda(\bar{r}) \rightarrow 0
$$

для всех $\bar{r} \in R^{\prime}(q, m)$, является необходимым и достаточным для асимптотической независимости случайных величин $\mu\left(B_{1}\right), \ldots, \mu\left(B_{m}\right)$.

Замечание 3. Множество $B$ назовем $r$-слойным, если каждая (проходящая через нуль) прямая, пересекающаяся с множеством $B$, имеет с ним ровно $r$ общих точек. При $K=G F(2)$ любое множество $B$, не содержащее нулевой точки, является однослойным. Если однослойные множества $B_{1}, \ldots, B_{m} \subseteq V^{T}$ попарно не пересекаются, то $\Lambda(\bar{r})=0$ для всех $\bar{r} \in R^{\prime}(q, m)$. Поэтому для них выполнено условие (7).

Задачи о числе ненулевых векторов в пересечении $L \cap B$ решаются путем сведения их к аналогичным задачам для числа решений соотношения (случайного линейного включения) $A x \in B$, где $x \in V^{n}, B \subseteq V^{T}$, а $A$ - случайная $T \times n$ матрица, составленная из независимых случайных величин, распределенных равновероятно на множестве элементов поля $K$. Используемые при этом результаты сформулированы в следующем разделе. Разделы 3 и 4 посвящены их доказательствам.

\section{2. Задача в терминах системы линейных уравнений}

Рассмотрим систему случайных уравнений над полем $G F(q)$

$$
a_{t, 1} x_{1} \oplus \ldots \oplus a_{t, n} x_{n}=b_{t}, \quad t=1, \ldots, T,
$$

относительно неизвестных $x_{1}, \ldots, x_{n}$. Коэффициенты $a_{t, i}$ линейных функций в левой части системы (8) предполагаются независимыми в совокупности случайными величинами, распределенными равномерно на $G F(q)$. Поэтому все уравнения в системе являются случайными. Случайными являются множество $S(b)$ и число $v(b)$ решений системы (8). 
Здесь и далее $b=\left(b_{1}, \ldots, b_{T}\right)$ - вектор правых частей уравнений системы $(8)$, рассматриваемый как вектор-столбец.

С традиционной проблематикой систем случайных линейных уравнений над конечным полем можно ознакомиться по работам [1]-[3]. Мы же рассмотрим следующую задачу. Пусть задано некоторое множество $B$ ненулевых правых частей системы (8). Рассмотрим множества $S(b)$ решений системы (8) при одной и той же левой части (в матричной форме записываем ее как $A x$ ), но разных правых частях из $B$. Нас интересует множество

$$
S(B)=\bigcup_{b \in B} S(b)
$$

и число его элементов $v(B)$.

Оно имеет простой и наглядный смысл. Это множество наборов $\left(x_{1}, \ldots, x_{n}\right)$, удовлетворяющих соотношению

$$
A x \in B \text {. }
$$

Соотношение вида (9) будем называть случайным линейным включением.

Пусть задан набор множеств $B_{1}, \ldots, B_{m} \subset V^{T}$. Используя обозначения предыдущего раздела, определим множества

$$
B(\bar{r})=\bigcup_{k \in F(\bar{r})} E_{T, k}
$$

Очевидно, что набор множеств $\{B(\bar{r})\}$ образует разбиение множества

$$
B^{\prime}=\bigcup_{\bar{r} \in R(q, m)} B(\bar{r})
$$

на непересекающиеся подмножества.

Следующее утверждение описывает асимптотические свойства набора случайных величин $\nu(B(\bar{r})), \bar{r} \in R(q, m)$, представляющих собой количества решений соотношений $A x \in B(\bar{r})$ при одной и той же случайной матрище $A$.

Пусть величины $\Lambda(\bar{r})$ определяются формулой (1). Заметим, что

$$
\Lambda(\bar{r})=\mathbf{E} v(B(\bar{r})) \text {. }
$$

Теорема 2. Пусть параметры $n$ и $T$ стремятся $\kappa$ бесконечности, а множества $B_{1}, \ldots, B_{m} \subseteq V^{T}$ меняются так, что выполнены условия (3) $u$ (4) $u$ для $B^{\prime}$ выполнено условие (5).

Тогда случайные величины $v(B(\bar{r})), \bar{r} \in R(q, m)$, асимптотически независимы и имеют в качестве предельных распределения Пуассона с параметрами $\lambda(\bar{r})$ соответственно. При этом сходимость распределения вектора $(\nu(B(\bar{r})), \bar{r} \in R(q, m))$ к распределению вектора $\left(\pi_{\bar{r}}(\lambda(\bar{r})), \bar{r} \in R(q, m)\right)$ равномерна для тех наборов множеств $B_{1}, \ldots, B_{m}$, которым отвечают одни и те же значения параметров $\Lambda(\bar{r})$.

Следствие 2. Пусть выполнены условия теоремы 2. Тогда распределение случайного вектора $\left(\nu\left(B_{1}\right), \ldots, v\left(B_{m}\right)\right)$, сходится к распределению случайного вектора (6).

Доказательство теоремы 2 приводится в следующем разделе. А сейчас мы воспользуемся этим результатом, чтобы доказать теорему, сформулированную во введении.

Рассмотрим величины $\mu(B(\bar{r}))=|L \cap B(\bar{r})|, \bar{r} \in R(q, m)$. 
Лемма 1. Случайные величины $\mu(B(\bar{r})), v(B(\bar{r})), \bar{r} \in R(q, m)$ могут быть заданы на одном вероятностном пространстве так, что

$$
\mathbf{P}\{\mu(B(\bar{r}))=v(B(\bar{r})), \bar{r} \in R(q, m)\} \rightarrow 1
$$

npu $T-n \rightarrow \infty$.

Доказательство. Рассмотрим случайное линейное подпространство $A V^{n}-$ образ $V^{n}$ в $V^{T}$ при случайном отображении, определяемом матрицей $A$. Заметим, что

$$
v(B(\bar{r}))=q^{s(A)}\left|A V^{n} \cup B(\bar{r})\right|,
$$

где $s(A)=n-\operatorname{rank}(A)$ и использован тот факт, что множества $B(\bar{r})$ не содержат нулевой вектор.

При $\operatorname{rank}(A)<n$, взяв произвольный базис в $A V^{n}$, достроим его до базиса из $n$ векторов путем случайного равновероятного выбора $s(A)$ недостающих линейно независимых векторов. Рассмотрим $n$-мерное линейное пространство $L_{A}$, натянутое на построенный базис.

Так как распределение матрицы $A$ инвариантно относительно любых невырожденных преобразований строк, случайное подпространство $L_{A}$ имеет на множестве всех $n$-мерных подпространств пространства $V^{T}$ равномерное распределение. Поэтому распределение набора случайных величин $(\mu(B(\bar{r})), \bar{r} \in R(q, m))$ совпадает с распределением набора $\left(\left|L_{A} \cap B(\bar{r})\right|, \bar{r} \in R(q, m)\right)$. С учетом равенства (12) это дает неравенство

$$
\mathbf{P}\{\mu(B(\bar{r}))=\nu(B(\bar{r})), \bar{r} \in R(q, m)\} \geqslant \mathbf{P}\{\operatorname{rank}(A)=n\} .
$$

Осталось заметить, что $\mathbf{P}\{\operatorname{rank}(A)=n\} \rightarrow 1$ при $T-n \rightarrow \infty$. Лемма доказана.

Лемма 2. Из условий теоремы 1 вытекает, что $T-n \rightarrow \infty$.

Доказательство. Из условий (3) и (4) теоремы 1 следует, что $q^{n-T}|F(\bar{r})| \leqslant C_{1}$ при некоторой константе $C_{1}$. Кроме этого, из определений вытекает, что

$$
|B| \leqslant(q-1) \sum_{\bar{r} \in R(q, m)}|F(\bar{r})| .
$$

Поэтому $q^{n-T}|B| \leqslant C_{2}$ при некоторой константе $C_{2}$. Осталось заметить, что $|B| \rightarrow \infty$ в силу условия (5) для $B$. Лемма 2 доказана.

С помощью лемм 1 и 2 получаем следующее утверждение для величин

$$
\mu(B(\bar{r}))=|L \cap B(\bar{r})|
$$

где $\bar{r} \in R(q, m)$, а подпространство $L$ выбрано случайно и равновероятно из множества всех $n$-мерных линейных подпространств пространства $V^{T}$.

Следствие 3. Пусть выполнены условия теоремы 2. Тогда случайные величины $\mu(B(\bar{r}))$, $\bar{r} \in R(q, m)$, асимптотически независимы и имеют в качестве предельных распределения Пуассона с параметрами $\lambda(\bar{r})$ соответственно. 
С помощью этого утверждения уже нетрудно доказать теорему 1. Заметим, что условия следствия 3 почти полностью совпадают с условиями теоремы 1. Надо лишь проверить, что

$$
N\left(B^{\prime}\right)\left|B^{\prime}\right|^{-2} \rightarrow 0
$$

Соотношение (13) следует из условия (5) для $B=\bigcup_{i} B_{i}$, включения $B^{\prime} \subseteq B$ и очевидных неравенств

$$
N\left(B^{\prime}\right) \leqslant N(B), \quad|B| \leqslant(q-1)\left|B^{\prime}\right| .
$$

Из следствия 3 и равенств

$$
\mu\left(B_{j}\right)=\sum_{\bar{r} \in R(q, m)} r_{j} \mu(B(\bar{r})), \quad \bar{r} \in R(q, m), \quad j=1, \ldots, m,
$$

вытекает утверждение теоремы 1.

\section{3. Доказательство теоремы 2}

Рассмотрим систему случайных индикаторов

$$
\eta_{x, b}=I\{A x=b\}, \quad(x, b) \in \Gamma,
$$

где

$$
\Gamma=\left\{(x, b): x \in V^{n} \backslash\left\{0^{n}\right\}, b \in B^{\prime}\right\}
$$

Очевидно, что

$$
\Gamma=\bigcup_{\bar{r} \in R(q, m)} \Gamma_{\bar{r}}, \quad \Gamma_{\bar{r}}=\left\{(x, b): x \in V^{n} \backslash\left\{0^{n}\right\}, b \in B(\bar{r})\right\},
$$

a

$$
v(B(\bar{r}))=\sum_{(x, b) \in \Gamma_{\bar{r}}} \eta_{x, b}
$$

Воспользуемся теоремой 1 работы [7], согласно которой указанные в теореме 2 асимптотические свойства вектора $(\nu(B(\bar{r})), \bar{r} \in R(q, m))$ имеют место, если выполнены следующие условия:

$$
\begin{aligned}
\sum_{(x, b) \in \Gamma_{\bar{r}}} \mathbf{E} \eta_{x, b} \rightarrow \lambda(\bar{r}), \quad k=1, \ldots, q-1, \\
\max _{(x, b) \in \Gamma} \mathbf{E} \eta_{x, b} \rightarrow 0,
\end{aligned}
$$

и при всех $r=2,3, \ldots$

$$
\sum\left|\mathbf{E} \eta_{x^{1}, b^{1}} \ldots \eta_{x^{r}, b^{r}}-\mathbf{E} \eta_{x^{1}, b^{1}} \ldots \mathbf{E} \eta_{x^{r}, b^{r}}\right| \rightarrow 0
$$

где суммирование проводится по множеству $I_{r}$ всех наборов из $r$ попарно различных элементов множества $\Gamma$. 
Замечание 4. Рассуждая от противного, нетрудно убедиться, что равномерная сходимость в соотношениях (16)-(18) (относительно наборов множеств $B_{1}, \ldots, B_{m}$, которым отвечают одни и те же значения параметров $\Lambda(\bar{r})$ ) гарантирует равномерную в том же смысле сходимость к предельному распределению для случайного вектора $(\nu(B(\bar{r})), \bar{r} \in R(q, m))$.

Замечание 5. Идея использовать условия типа (16)-(18) в качестве достаточных условий сходимости распределения суммы зависимых случайных индикаторов к пуассоновскому принадлежит Б. А. Севастьянову. Формулировку и доказательство теоремы Севастьянова в одномерном случае (то есть только для одной суммы индикаторов) можно найти в статье [8] и монографии [9]. Условия (16)-(18) несколько отличаются от условий Б. А. Севастьянова, но, как было показано в работе [10], в одномерном случае эквивалентны им. Доказательство используемой нами многомерной версии теоремы Севастьянова приведено в [10]. Оно отличается от доказательства одномерного варианта лишь в деталях.

Перейдем к проверке свойств (16)-(18). Первые два из них следуют непосредственно из условий теоремы 2. Поэтому сразу обратимся к проверке условия (18).

Разобьем множество $I_{r}, r \geqslant 2$, в объединение непересекающихся множеств

$$
I_{r}=J_{r} \cup I_{r, r}=I_{r, 1} \cup \ldots \cup I_{r, r},
$$

где

$$
I_{r, k}=\left\{\left(\left(x^{1}, b^{1}\right), \ldots,\left(x^{r}, b^{r}\right)\right) \in I_{r}: \operatorname{rank}\left(x^{1}, \ldots, x^{r}\right)=k\right\} .
$$

При условии, что $\operatorname{rank}\left(x^{1}, \ldots, x^{r}\right)=r$, выполнены равенства

$$
\mathbf{E} \eta_{x^{1}, b^{1}} \ldots \eta_{x^{r}, b^{r}}=\mathbf{E} \eta_{x^{1}, b^{1}} \ldots \mathbf{E} \eta_{x^{r}, b^{r}}=q^{-r T}
$$

Поэтому слагаемые суммы (18), отвечающие индексам из множества $I_{r, r}$, равны нулю. Следовательно, для проверки (18) достаточно показать, что любого фиксированного $r=2,3, \ldots$

$$
\begin{aligned}
T_{r} & =\sum_{J_{r}} \mathbf{E} \eta_{x^{1}, b^{1}} \ldots \mathbf{E} \eta_{x^{r}, b^{r}} \rightarrow 0, \\
S_{r} & =\sum_{J_{r}} \mathbf{E} \eta_{x^{1}, b^{1}} \ldots \eta_{x^{r}, b^{r}} \rightarrow 0 .
\end{aligned}
$$

Условие (21) проверяется довольно просто. Так как $\mathbf{E} \eta_{x, b}=q^{-T}$, то

$$
T_{r}=\left(q^{-T}\right)^{r}\left|J_{r}\right|=\left(q^{-T}\right)^{r}\left(\left(q^{n}-1\right)^{r}-\prod_{s=0}^{r-1}\left(q^{n}-q^{s}\right)\right)\left|B^{\prime}\right|^{r}
$$

Из условий теоремы следует, что выражение в правой части этой цепочки равенств стремится к нулю.

Заметим, что слагаемые в (22), для которых

$$
a_{1} x^{i_{1}} \oplus \ldots \oplus a_{s} x^{i_{s}}=0^{n}, \quad a_{1} b^{i_{1}} \oplus \ldots \oplus a_{s} b^{i_{s}} \neq 0^{T}
$$

при некоторых $a_{1}, \ldots, a_{s} \in K$, равны нулю. Поэтому $S_{2}=0$.

Для проверки условия (22) при $r \geqslant 3$ нам потребуется следующее утверждение. 
Лемма 3. При $3 \leqslant r \leqslant n$ выполнено неравенство

$$
S_{r} \leqslant \frac{N\left(B^{\prime}\right)}{\left|B^{\prime}\right|^{2}}\left(\Lambda^{\prime}+q^{r-1}-2 q+3\right)^{r} .
$$

Используя лемму 3, завершаем проверку условия (22). Остается отметить, что все полученные оценки равномерны в указанном в замечании 4 смысле. Теорема 2 доказана.

Доказательство леммы 3. Каждому набору векторов $x^{1}, \ldots, x^{r} \in V^{n} \backslash\left\{0^{n}\right\}$ соответствует система $L_{x}\left(x^{1}, \ldots, x^{r}\right)$ однородных линейных уравнений в $V^{n}$ вида

$$
a_{1} x^{i_{1}} \oplus \ldots \oplus a_{s} x^{i_{s}}=0^{n}
$$

(возможно пустая), состоящая из всех линейных соотношений, которым удовлетворяет набор векторов $x^{1}, \ldots, x^{r}$. Системе $L_{x}\left(x^{1}, \ldots, x^{r}\right)$ сопоставим идентичную по записи систему $L_{b}\left(x^{1}, \ldots, x^{r}\right)$ из линейных уравнений относительно $b^{1}, \ldots, b^{r} \in B$. Обозначим через $B\left(x^{1}, \ldots, x^{r}\right)$ множество решений $\left(b^{1}, \ldots, b^{r}\right) \in B^{r}$ системы уравнений $L_{b}\left(x^{1}, \ldots, x^{r}\right)$. Тогда

$$
S_{r} \leqslant \sum_{k=2}^{r-1} \sum_{\left(x^{1} \ldots, x^{r}\right) \in I_{r, k}} \sum_{B\left(x^{1} \ldots . x^{r}\right)} \mathbf{E} \eta_{x^{1}, b^{1}} \ldots \eta_{x^{r}, b^{r}},
$$

где

$$
X_{r, k}=\left\{\left(x_{1}, \ldots, x_{r}\right): x_{1}, \ldots, x_{r} \in V^{T} \backslash\left\{0^{T}\right\}, \operatorname{rank}\left(x^{1}, \ldots, x^{r}\right)=k\right\} .
$$

Нетрудно проверить, что

$$
\mathbf{E} \eta_{x^{1}, b^{1}} \ldots \eta_{x^{r}, b^{r}} \leqslant q^{-k T}
$$

если $\operatorname{rank}\left(x^{1}, \ldots, x^{r}\right)=k$. Поэтому из (25) при $n \geqslant r \geqslant 3$ вытекает оценка

$$
S_{r} \leqslant \sum_{k=2}^{r-1} \sum_{\left(x^{1}, \ldots, x^{r}\right) \in X_{r, k}} \frac{\left|B\left(x^{1}, \ldots, x^{r}\right)\right|}{q^{k T}} .
$$

Из определения величины $N(B)$ вытекает следующее утверждение. Обозначим через $N\left(r, a_{1}, \ldots, a_{r}, d\right)$, где $a_{1}, \ldots, a_{r} \in G F(q) \backslash\{0\}, d \in V^{T}$, число решений уравнения

$$
a_{1} u^{1} \oplus \ldots \oplus a_{r} u^{r}=d
$$

относительно набора неизвестных $\left(u^{1}, \ldots, u^{r}\right), u^{1}, \ldots, u^{r} \in B^{\prime}$.

Лемма 4. При $r \geqslant 3$ и любых $a_{1}, \ldots, a_{r} \in K \backslash\{0\}, d \in V^{T}$

$$
N\left(r, a_{1}, \ldots, a_{r}, d ; B\right) \leqslant\left|B^{\prime}\right|^{r-3} N\left(B^{\prime}\right) .
$$

Утверждение леммы 4 очевидно.

Лемма 5. Пусть $\operatorname{rank}\left(x^{1}, \ldots, x^{r}\right)=k u 2 \leqslant k \leqslant r-1$. Тогда

$$
\left|B\left(x^{1}, \ldots, x^{r}\right)\right| \leqslant\left|B^{\prime}\right|^{k-2} N\left(B^{\prime}\right) .
$$


Доказательство лемиы 5. Перенумеруем векторы $x^{1}, \ldots, x^{r}$ так, чтобы первые $k$ из них составляли базис. Тогда систему $L_{b}\left(x^{1}, \ldots, x^{r}\right)$ можно преобразовать к виду

$$
\begin{aligned}
b^{k+1} & =l_{k+1}\left(b^{1}, \ldots, b^{k}\right), \\
& \ldots \\
b^{r} & =l_{r}\left(b^{1}, \ldots, b^{k}\right)
\end{aligned}
$$

с некоторыми линейными функциями $l_{k+1}, \ldots, l_{r}$ своих аргументов. Так как из этих уравнений значения $b^{k+2}, \ldots, b^{r} \in B^{\prime}$ определяются величинами $b^{1}, \ldots, b^{k}$ одюозначно, число решений системы (26) относительно $b^{1}, \ldots, b^{r} \in B^{\prime}$ не превосходит числа решений уравнения $b^{k+1}=l_{k+1}\left(b^{1}, \ldots, b^{k}\right)$ относительно $b^{1}, \ldots, b^{k+1}$. Последнее, как это следует из леммы 3 , не превосходит $\left|B^{\prime}\right|^{k-2} N\left(B^{\prime}\right)$, если только число $s$ существенных аргументов в выражении $\left.b^{k+1} \ominus l_{k+1}\left(b^{1}, \ldots, b^{k}\right)\right)$ удовлетворяет неравенству $s \geqslant 3$. Последнее неравенство имеет место, поскольку в системах $L_{x}\left(x^{1}, \ldots, x^{r}\right)$ и $L_{b}\left(x^{1}, \ldots, x^{r}\right)$ нет одночленных и двучленных уравнений. Лемма 5 доказана.

Вернемся к неравенству (26). Воспользуемся в нем оценкой (28), что дает неравенство

$$
S_{r} \leqslant \sum_{k=2}^{r-1}\left|X_{r, k}\right| \frac{\left|B^{\prime}\right|^{k-2} N\left(B^{\prime}\right)}{q^{k T}},
$$

и очевидными оценками

$$
\left|X_{r, k}\right| \leqslant\left(\begin{array}{l}
r \\
k
\end{array}\right)\left(q^{k}-k(q-2)-1\right)^{r-k} \prod_{s=0}^{k-1}\left(q^{n}-q^{s}\right) .
$$

После несложных преобразований получаем (24). Лемма 3 доказана.

\section{4. Заключительные замечания}

В качестве еще одного примера применения теоремы 2 рассмотрим задачу о числе приближенных решений однородной системы случайных линейных уравнений $A x=0$, a именно, задачу о числе таких векторов $x$, при которых система $A x=0$ нарушается ровно (или, в ином варианте, не более, чем) в $w$ уравнениях.

Условимся использовать обозначение $\|b\|$ для числа ненулевых среди координат вектора $b$. Пусть

$$
\begin{aligned}
& B(w)=\left\{b \in V^{T}:\|b\|=w\right\}, \quad w \geqslant 1, \\
& C(w)=\left\{b \in V^{T}: 1 \leqslant\|b\| \leqslant w\right\} .
\end{aligned}
$$

Очевидно, что

$$
|B(w)|=\left(\begin{array}{l}
T \\
w
\end{array}\right)(q-1)^{w}, \quad|C(w)|=\sum_{k=1}^{w}\left(\begin{array}{l}
T \\
k
\end{array}\right)(q-1)^{k} .
$$

Заметим, что вместе с любым вектором $b \in B(w)$ в множество $B(w)$ входят все коллинеарные ему векторы. Этим же свойством обладает и множество $C(w)$. Поэтому носители распределения случайных величин $v(B(\omega))$ и $v(C(\omega))$ сосредоточены на решетке $\{(q-1) k, k=0,1, \ldots\}$.

Используя следствие 2, можно доказать следующее утверждение. 
Теорема 3. Пусть параметры п и $T$ стремятся к бесконечности, а параметр $w \geqslant 1$ меняется так, что $w / T \rightarrow 0 u$

$$
\left(\begin{array}{l}
T \\
w
\end{array}\right) q^{n-T}(q-1)^{w-1} \rightarrow \lambda, \quad 0<\lambda<\infty .
$$

Тогда распределения случайных величин

$$
(q-1)^{-1} v(B(w)), \quad(q-1)^{-1} v(C(w))
$$

сходятся к распределению Пуассона с параметром $\lambda$.

Замечание 6. В условиях теоремы 3 выполнено соотношение

$$
\mathbf{P}\{v(B(w)=v(C(w)))\} \rightarrow 1 .
$$

\section{Список литературы}

1. Колчин В. Ф., Системы случайных уравнений. МИЭМ, Москва, 1988.

2. Колчин В. Ф., Случайные графы. Наука, Москва, 2000.

3. Балакин Г. В., Системы случайных уравнений над конечным полем. Труды по дискретной математике (1998) 2, 21-37.

4. Михайлов В. Г., Предельные теоремы для числа ненулевых решений одной системы случайных уравнений над полем $G F(2)$. Теория вероятностей и ее применения (1998) 43, №3, 598-606.

5. Михайлов В. Г., Предельные теоремы для числа ненулевых решений одной системы случайных уравнений над полем $G F(2)$. Дискретная математика (2000) 12, №1, 70-81.

6. Михайлов В. Г., Предельная теорема Пуассона для числа неколлинеарных решений системы случайных уравнений специального вида. Дискретная математика (2001) 13, №3, 81-90.

7. Михайлов В. Г., Сходимость к процессу с независимыми приращениями в схеме нарастающих сумм зависимых случайных величин. Матем. сб. (1974) 94, №2, 283-299.

8. Севастьянов Б. А., Предельный закон Пуассона в схеме сумм зависимых случайных величин. Теория вероятностей и ее применения (1972) 17, №4, 733-738.

9. Колчин В. Ф., Севастьянов Б. А., Чистяков В. П., Случайные размещения. Наука, Москва, 1976.

10. Михайлов В. Г., Некоторые предельные теоремы для сумм зависимых случайных величин. Дисс. на соиск. степени канд. физ.-матем. наук, МИАН, Москва, 1974.

Статья поступила 08.01.2003. 